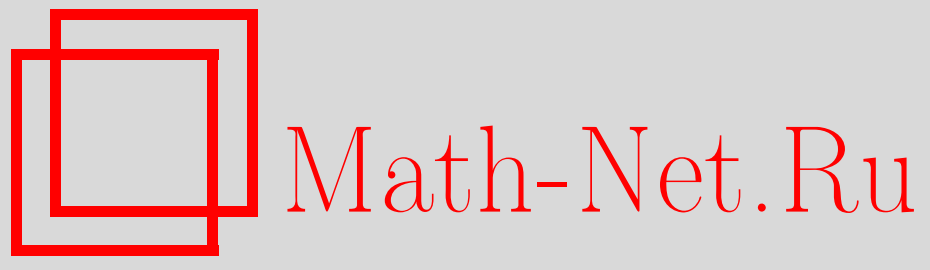

И. С. Борисов, Замечание о распределении числа пересечений полосы случайным блужданием, Теория вероятн. и ее примен., 2008, том 53, выпуск 2, 345-349

DOI: https://doi.org/10.4213/tvp2413

Использование Общероссийского математического портала Math-Net.Ru подразумевает, что вы прочитали и согласны с пользовательским соглашением

http: //www . mathnet.ru/rus/agreement

Параметры загрузки:

IP: 107.22 .136 .117

26 апреля 2023 г., $07: 40: 53$

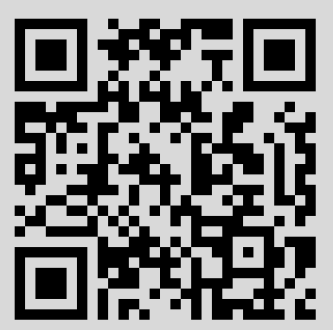


(C) $2008 \Gamma$

БОРИСОВ И. С. *

\section{ЗАМЕЧАНИЕ О РАСПРЕДЕЛЕНИИ ЧИСЛА ПЕРЕСЕЧЕНИЙ ПОЛОСЫ СЛУЧАЙНЫМ БЛУЖДАНИЕМ ${ }^{1)}$}

В работе сушественно обобшается результат из [1], где была приведена формула для распределения числа пересечений полосы траекторией случайного блуждания, порожденного бесконечной последовательностью частичных сумм независимых случайных величин с одним и тем же «двусторонним геометрическим» распределением.

Ключевые слова и фразы: случайное блуждание, процесс частичных сумм, марковское свойство перескока.

Пусть $\left\{\xi_{i} ; i \geqslant 1\right\}$ - последовательность независимых одинаково распределенных случайных величин с условиями

$$
\mathbf{E} \xi_{1} \neq 0, \quad \mathbf{P}\left\{\xi_{1}>0\right\} \mathbf{P}\left\{\xi_{1}<0\right\} \neq 0 .
$$

Определим процесс частичных сумм на положительной полупрямой по формуле

$$
S(t):=\sum_{1 \leqslant i \leqslant t} \xi_{i}, \quad t \geqslant 0,
$$

где сумма по пустому множеству индексов по определению полагается равной нулю. Этот случайный процесс описывает однородное по времени случайное блуждание частицы на числовой прямой с независимыми скачками $\left\{\xi_{i}\right\}$, происходяшими через единичные промежутки времени.

Обозначим через $\eta_{1}$ и $\eta_{2}$ соответственно число пересечений сверху вниз и снизу вверx (более формально эти определения даны при доказательстве нижеследующей теоремы) траекторией $S(\cdot)$ полосы

$$
D(N, M):=\{(t, s): t \geqslant 0,-M \leqslant s \leqslant N\},
$$

где $N, M>0$. Для блужданий с целочисленными скачками дополнительно предполагается, что $N$ и $M$ - натуральные числа. Отметим, что при выполнении (1) случайные величины $\eta_{1}$ и $\eta_{2}$ будут собственными, поскольку в этом случае рассматриваемое случайное блуждание с вероятностью 1 будет иметь ненулевой снос на бесконечность того или иного знака.

Поводом для написания настоящей заметки послужила статья [1], в которой было доказано, что если

$$
\mathbf{P}\left\{\xi_{1}=k\right\}=a_{1} q_{1}^{k}, \quad \mathbf{P}\left\{\xi_{1}=-k\right\}=a_{2} q_{2}^{k}, \quad k=1,2, \ldots,
$$

то для всех натуральных $k$

$$
\mathbf{P}\left\{\eta_{1} \geqslant k\right\}=c_{1} d^{k}, \quad \mathbf{P}\left\{\eta_{2} \geqslant k\right\}=c_{2} d^{k},
$$

где параметры $c_{i}$ и $d$ определяются распределением (2). При этом использовался достаточно громоздкий аналитический аппарат, основанный на представлении искомых вероятностей через компоненты факторизации функции двух комплексных переменных $1-z \varphi(\lambda)$, где $\varphi(\lambda)$ - преобразование Лапласа распределения $\xi_{1}$.

Цель настояшей заметки - привести элементарное доказательство утверждения (3) для значительно более широкого, чем (2), класса распределений $\xi_{1}$. При этом будет дана вероятностная интерпретация постоянных $c_{i}$ и $d$.

* Институт математики им. С. Л. Соболева СО РАН, просп. Академика Коптюга, 4, 630090 Новосибирск, Россия; e-mail: sibam@math.nsc.ru

1) Работа выполнена при поддержке РФФИ (гранты 05-01-00810, 06-01-00738) и INTAS (грант 03-51-5018). 
Отметим, что при выполнении (1) распределение случайной величины $\xi_{1}$ может быть представлено единственным образом в виде смеси распределений положительной компоненты $\xi_{1}^{+}$и жеположительной компоненты $\xi_{1}^{-}$, т.е. справедливо равенство по распределению

$$
\xi_{1} \stackrel{d}{=} \nu \xi_{1}^{+}+(1-\nu) \xi_{1}^{-},
$$

где $\nu$ - бернуллиевская случайная величина с некоторой вероятностью успеха (равной весу упомянутой смеси), $\xi_{1}^{+}>0$ и $\xi_{1}^{-} \leqslant 0$ почти наверное, причем все три случайные величины $\xi_{1}^{+}, \xi_{1}^{-}$и $\nu$ предполагаются независимьими.

Мы сохраним термины положительная и неположительная компоненты скачка распределения и в случае, когда

$$
\xi_{1} \stackrel{d}{=} \xi_{1}^{+}+\xi_{1}^{-}
$$

если только эти компоненты независимы. Понятно, что представление (5), нередко встречающееся в приложениях (например, в теории массового обслуживания), может быть переписано в виде (4), но с другими компонентами. Но в некоторых случаях те или иные компоненты в (4) и (5) могут совпадать. Например, скачок любого полунепрерывного сверху случайного блуждания допускает указанные представления с одной и той же вырожденной положительной компонентой $\xi_{1}^{+}$, равной единице почти наверное. При этом неположительные компоненты могут различаться: в отличие от (4) в представлении (5) в рассматриваемом примере случайная величина $\xi_{1}^{-}$, распределенная на множестве неположительных целых чисел, в силу (1) с необходимостью должна удовлетворять условию $\mathbf{P}\left\{\xi_{1}^{-}=0\right\}>0$.

Мы будем говорить, что случайная величина $\zeta$ обладает марковским перескоком, если при всех тех $x>0$ из носителя ее распределения, для которых $\mathbf{P}\{\zeta \geqslant x\}>0$, условное распределение разности (величины перескока) $\zeta-x$ при условии $\zeta \geqslant x$ не зависит om $x$. Ясно, что в силу очевидной симметрии рассматриваемой задачи мы можем ограничиться только изучением перескока вправо, при необходимости применяя вышеприведенное определение к случайной величине $-\zeta$. Хорошо известно, что указанным свойством обладают экспоненциальное и геометрическое распределения (см., например, [2]), поскольку эффект отсутствия последействия у распределения перескока (т.е. совпадение распределения исходной случайной величины с соответствующим условным распределением величины ее перескока через любой уровень) для этих двух семейств распределений влечет за собой отмеченное марковское свойство (но не наоборот!). Очевидно, этим же свойством обладают и вырожденные распределения на положительной полупрямой, а также любые смеси экспоненциального (или геометрического) распределения с вырожденным в нуле распределением. Более того, с помощью формулы полной вероятности можно легко убедиться в том, что марковский перескок имеют случайные величины, представимые в виде (4) или (5), если только положительная компонента обладает указанным свойством, а неположительная имеет произвольное распределение при условии согласования их носителей. Так что, говоря в нижеследующей теореме о марковском перескоке скачка случайного блуждания при выполнении (1), мы будем иметь в виду следуюшие три типа распределений $\xi_{1}$ :

(I) положительная компонента $\xi_{1}^{+}$имеет геометрическое распределение (на множестве натуральных чисел), а $\xi_{1}^{-}$произвольно распределена на множестве чельх неположительных чисел;

(II) $\xi_{1}^{+}=1$ почти наверное, а $\xi_{1}^{-}$имеет произвольное распределение на множестве целых неположительных чисел (полунепрерывное случайное блуждание);

(III) $\xi_{1}^{+}$экспоненциально распределена, а $\xi_{1}^{-}$имеет произвольное распределение с носителем на замкнутой справа полуоси $(-\infty, 0]$.

Отметим, что класс (III) содержит все распределения с непрерывной положительной компонентой и марковским перескоком. Классы (I) и (II) с точностью до шага и сдвига решетки также описывают все дискретные распределения (которые, как нетрудно понять, будут с необходимостью решетчатыми) с указанным свойством перескока. Доказательства этих свойств по сути повторяют соответствующие рассуждения в [2], и поэтому мы их опускаем.

Основной результат настояшей заметки состоит в следующем. 
Теорема. Пусть выполнено условие (1) и распределение скачка $\xi_{1}$ (или - $\left.\xi_{1}\right)$ случайного блуждания $S(t)$ принадлежит одному из классов (I)-(III). Тогда справедливы оба соотношения в (3).

Д о к а з а т е л ь с т в о. В силу очевидной симметрии задачи ограничимся рассмотрением случая, когда распределение $\xi_{1}$ принадлежит одному из классов (I)(III). Прежде всего введем необходимые обозначения. На множестве траекторий $S(\cdot)$, пересекающих уровень $N>0$, определим

$$
\tau_{1}^{+}:=\min \{t \geqslant 0: S(t) \geqslant N\}, \quad S_{1}(t):=S\left(\tau_{1}^{+}+t\right)-S\left(\tau_{1}^{+}\right), \quad t \geqslant 0 .
$$

На оставшихся элементарных исходах (траекториях) положим $\tau_{1}^{+}=\infty$ и $S(\infty)=0$. Построенные отображения вероятностного пространства на расширенную числовую прямую (с расширенной борелевской $\sigma$-алгеброй) будут измеримыми, и мы вправе говорить о них как о случайных величинах. Теперь положим $\chi_{1}^{+}:=\max \left\{S\left(\tau_{1}^{+}\right), N\right\}-N$. Такие случайные величины иногда называются лестничньими высотами (см. [2, т. 2], [3]), которые для краткости мы также будем называть перескоками (через уровень $N$ ).

Обозначим через $\mathscr{B}(k), k=1,2, \ldots, \sigma$-алгебру событий, порожденную траекториями $S(\cdot)$ до момента $t=k$ включительно, а через $\mathscr{B}\left(\tau_{1}^{+}\right)-$минимальную $\sigma$-алгебру, содержашую события вида $B \cap\left\{\tau_{1}^{+}=k\right\}$ при всех $B \in \mathscr{B}(k)$ и $k<\infty$. Кроме того, пусть $\mathscr{B}_{1}$ обозначает $\sigma$-алгебру событий, порожденных траекториями случайного процесса $S_{1}(t)$ при $t \geqslant 0$. Наконец, положим $\mathscr{P}(A):=\mathbf{P}\left\{\xi_{1}-1 \in A \mid \xi_{1} \geqslant 1\right\}$. На основном вероятностном пространстве зададим также случайную величину $\chi^{*}$ (собственный nерескок) с распределением $\mathscr{P}(A)$, не зависящую от последовательности $\left\{\xi_{i}\right\}$.

$\mathrm{C}$ помошью формулы полной вероятности (при фиксации момента перескока и положения траектории $S(\cdot)$ в момент, предшествуюший перескоку) и марковского свойства перескока у скачка рассматриваемого случайного блуждания элементарно доказывается следуюшее утверждение.

Лемма. Пусть выполнены условия теоремы. Тогда для всех борелевских подмножеств $А$ неотрицательной полуоси справедливы следуюшие соотношения:

$$
\begin{aligned}
& \mathbf{P}\left\{\chi_{1}^{+} \in A \mid \tau_{1}^{+}=k\right\}=\mathscr{P}(A) \quad \text { при всех натуральньи } \quad k, \\
& \mathbf{P}\left\{B \cap\left\{\chi_{1}^{+} \in A\right\} \cap C\right\}=\mathbf{P}(B) \mathbf{P}(C) \mathscr{P}(A) \quad \text { nри всех } \quad B \in \mathscr{B}\left(\tau_{1}^{+}\right) \quad u \quad C \in \mathscr{B}_{1} .
\end{aligned}
$$

Далее, аналогично (6) определяем

$$
\begin{array}{ll}
\tau_{1}^{-}:=\min \left\{t>\tau_{1}^{+}: S(t) \leqslant-M\right\}, & \tau_{2}^{+}:=\min \left\{t>\tau_{1}^{-}: S(t) \geqslant N\right\}, \\
\tau_{2}^{-}:=\min \left\{t>\tau_{2}^{+}: S(t) \leqslant-M\right\}, & \chi_{2}^{+}:=\max \left\{S\left(\tau_{2}^{+}\right), N\right\}-N,
\end{array}
$$

где минимальный элемент пустого множества по-прежнему полагается равным $\infty$. Продолжая неограниченно этот процесс построения, мы зададим на одном вероятностном пространстве последовательности случайных величин (вообше говоря, несобственных) $\left\{\tau_{j}^{+}, \tau_{j}^{-} ; j \geqslant 1\right\}$, а также собственные случайные величины $\left\{\chi_{j}^{+}\right\}-$последовательные перескоки исходного случайного блуждания $S(t)$ через «верхний» уровень после очередного пересечения «нижнего». При этом первое утверждение леммы перепишется в виде

$$
\mathbf{P}\left\{\chi_{j}^{+} \in A \mid \tau_{j}^{+}=k\right\}=\mathbf{P}\left\{\chi^{*} \in A\right\}=\mathscr{P}(A) \quad \text { при всех натуральных } j \quad \text { и } k,
$$

а соответствуюший аналог второго утверждения леммы влечет следующие рекуррентные соотношения:

$$
\begin{gathered}
\mathbf{P}\left\{\tau_{j}^{-}<\infty\right\}=\mathbf{P}\left\{\tau_{j}^{+}<\infty\right\} \mathbf{P}\left\{\chi^{*}+\inf _{t \geqslant 0} S(t) \leqslant-N-M\right\}, \quad j \geqslant 1, \\
\mathbf{P}\left\{\tau_{j}^{+}<\infty\right\}=\mathbf{P}\left\{\tau_{j-1}^{+}<\infty\right\} \mathbf{P}(B), \quad j>1,
\end{gathered}
$$

где событие $B$ состоит в том, что траектории случайного прочесса $\chi^{*}+S(t) n p u$ $t \geqslant 0$ сначала пересекают уровень $-N-M$, а затем нулевой уровень. Очевидно, отсюда сразу следует соотношение

$$
\mathbf{P}\left\{\eta_{1} \geqslant k\right\}=\mathbf{P}\left\{\tau_{k}^{-}<\infty\right\}=c_{1} d^{k}, \quad k \geqslant 1,
$$


где $d:=\mathbf{P}(B)$ и $c_{1}:=\mathbf{P}\left\{\tau_{1}^{+}<\infty\right\} \mathbf{P}\left\{\chi^{*}+\inf _{t \geqslant 0} S(t) \leqslant-N-M\right\} / \mathbf{P}(B)$. Аналогичное распределение имеет и величина $\eta_{2}$, поскольку

$$
\begin{aligned}
\mathbf{P}\left\{\eta_{2} \geqslant k\right\}= & \mathbf{P}\left\{\tau_{1}^{+}<\infty, \inf _{0 \leqslant t \leqslant \tau_{1}^{+}} S(t)>-M\right\} \mathbf{P}(B)^{k} \\
& +\mathbf{P}\left\{\tau_{1}^{+}<\infty, \inf _{0 \leqslant t \leqslant \tau_{1}^{+}} S(t) \leqslant-M\right\} \mathbf{P}(B)^{k-1} .
\end{aligned}
$$

Так что в формуле для распределения $\eta_{2}$ в (3) нужно положить

$$
c_{2}:=\mathbf{P}\left\{\tau_{1}^{+}<\infty, \inf _{0 \leqslant t \leqslant \tau_{1}^{+}} S(t)>-M\right\}+\frac{\mathbf{P}\left\{\tau_{1}^{+}<\infty, \inf _{0 \leqslant t \leqslant \tau_{1}^{+}} S(t) \leqslant-M\right\}}{\mathbf{P}(B)} .
$$

Теорема доказана.

3 а м е ч а н и е 1 . В зависимости от знака $\mathrm{E} \xi_{1}$ (т.е. от направления сноса траектории $S(t)$ ) постоянные $c_{i}$ в приведенных формулах допускают уточнение. Скажем, если $\mathbf{E} \xi_{1}>0$, то событие $\left\{\tau_{1}^{+}<\infty\right\}$ будет достоверным (см. [2, т. 2], [3]) и его (а также его вероятность) следует удалить в формулах для $c_{i}$. Если же $\mathbf{E} \xi_{1}<0$, то в определении $c_{1}$ мы имеем $\mathbf{P}\left\{\chi^{*}+\inf _{t \geqslant 0} S(t) \leqslant-N-M\right\}=1$.

3 а м е ч а н и е 2. Если одновременно обе случайные величины $\xi_{1}$ и $-\xi_{1}$ обладают марковскими перескоками (скажем, как в (2)), то вычисление параметров $c_{i}$ и $d$ распределений в вышеприведенных формулах сводится к известным простым задачам о пересечении уровня и вариантам двуграничной «задачи о разорении» для рассматриваемого случайного блуждания. При этом соответствующие вероятности могут быть вычислены сравнительно элементарно без привлечения аппарата факторизационных тождеств (см. [3]) или теории восстановления (с использованием интегрального уравнения Винера-Хопфа, как, например, в [2, т. 2]).

В качестве примера продемонстрируем, как в условиях замечания 2 можно вычислить параметры $c_{1}$ и $d$. Прежде всего отметим, что в рассматриваемом случае величина $d$ (в силу упомянутых свойств перескоков вправо и влево) допускает следующее представление:

$$
d=\mathbf{P}\left\{\chi^{*}+\inf _{t \geqslant 0} S(t) \leqslant-N-M\right\} \mathbf{P}\left\{\chi_{-}^{*}+\sup _{t \geqslant 0} S(t) \geqslant N+M\right\}
$$

где случайная величина $\chi_{-}^{*}$ (собственный перескок влево), не зависящая от $\chi^{*}$ и случайного процесса $S(\cdot)$, определяется так же, как $\chi^{*}$, но для случайной величины $-\xi_{1}$. Иначе говоря, для вычисления компонент $c_{1}$ и $d$ нужно знать лишь вероятности пересечения уровня рассматриваемым случайным блужданием, что составляет содержание известных граничных задач. Наша цель - привести элементарный вывод уже известных соотношений (см., например, [2], [3]).

Мы ограничимся вычислением вероятности

$$
Q(x):=\mathbf{P}\left\{\sup _{t \geqslant 0} S(t) \geqslant x\right\} \quad \text { при любых } x>0
$$

к которой, очевидно, все сводится. При этом мы будем требовать марковское свойство перескока только у $\xi_{1}$, но не у $-\xi_{1}$, т.е. приводимые ниже рассуждения справедливы для любого представителя классов (I)-(III). Кроме того, мы уточним моментное условие в (1), предположив, что $\mathbf{E} \xi_{1}<0$, поскольку в противном случае, как уже было отмечено, $Q(x) \equiv 1$.

В самом деле, с помошью формулы полной вероятности и вышеприведенной леммы нетрудно получить при любых $x, \delta>0$ следующее тождество:

$$
Q(x+\delta)=Q(x)\left(\mathbf{P}\left\{\chi^{*} \geqslant \delta\right\}+\int_{[0, \delta)} Q(\delta-t) \mathbf{P}\left\{\chi^{*} \in d t\right\}\right) .
$$

Скажем, если распределение $\xi_{1}$ принадлежит классу (III), то из (8) легко выводим дифференциальное уравнение $Q^{\prime}(x)=\alpha\left(Q\left(0^{+}\right)-1\right) Q(x)$, где $Q\left(0^{+}\right):=$ $\lim _{x \rightarrow 0+0} Q(x)=\mathbf{P}\left\{\sup _{t \geqslant 0} S(t)>0\right\}$. Отсюда немедленно следует, что

$$
Q(x)=Q\left(0^{+}\right) \exp \left\{-\alpha\left(1-Q\left(0^{+}\right)\right) x\right\}
$$


при всех $x \geqslant 0$; здесь $\alpha$ - показатель экспоненциального распределения положительной компоненты $\xi_{1}^{+}$. Для вычисления величины $Q\left(0^{+}\right)$можно вновь воспользоваться формулой полной вероятности (с учетом экспоненциального представления (9)):

$$
1-Q\left(0^{+}\right)=\int_{-\infty}^{0} \mathbf{P}\left\{\xi_{1} \in d x\right\}(1-Q(|x|))=p-p Q\left(0^{+}\right) \varphi^{-}\left(\alpha\left(1-Q\left(0^{+}\right)\right)\right),
$$

где $p:=\mathbf{P}\left\{\xi_{1} \leqslant 0\right\}$, а $\varphi^{-}(s)$ - преобразование Лапласа распределения случайной величины $\left|\xi_{1}^{-}\right|$. Полагая $z:=\alpha\left(1-Q\left(0^{+}\right)\right)$, получаем из (10) уравнение для нахождения $Q\left(0^{+}\right)$.

$$
\varphi^{-}(z)=f(z)
$$

где $f(x):=p^{-1}-\left(p^{-1}-1\right)(1-z / \alpha)^{-1}$. Нетрудно видеть, что это уравнение в интервале $(0, \alpha)$ имеет единственное решение. Это следует из соотношений $\varphi^{-}(0)=f(0)=1,\left(\varphi^{-}(0)\right)^{\prime}=-\mathbf{E}\left|\xi_{1}^{-}\right|<f^{\prime}(0)=-\left(p^{-1}-1\right) / \alpha$, поскольку $\mathbf{E} \xi_{1}=$ $(1-p) / \alpha-p \mathbf{E}\left|\xi_{1}^{-}\right|<0$, а также того факта, что $\varphi^{-}(z)$ - положительная выпуклая функция, а $f(z)$ - вогнутая функция, меняющая свой знак при $z \rightarrow \alpha-0$. Отметим также, что уравнение (11) для нахождения $Q\left(0^{+}\right)$эквивалентно соответствуюшему уравнению в $[3$, с. 236], записанному в терминах преобразования Лапласа распределения скачка $\xi_{1}$. Основанное на методике теории восстановления вычисление функции $Q(x)$ для класса (III) также можно найти в [2, т. 2, с. 457].

Для арифметических распределений из классов (I) и (II) тождество (8) преврашается в простое рекуррентное соотношение

$$
Q(k+1)=Q(k)\left(\mathbf{P}\left\{\chi^{*} \geqslant 1\right\}+Q(1) \mathbf{P}\left\{\chi^{*}=0\right\}\right)
$$

для любого целого $k \geqslant 0$, которое также приводит к показательному распределению $Q(k)$ с уравнением для $Q(1)=Q\left(0^{+}\right)$, аналогичным (10). Отметим, что для полунепрерывного случайного блуждания (класс (II)) мы имеем $\mathbf{P}\left\{\chi^{*}=0\right\}=1$, и из (12) получаем, что $Q(k)=Q(1)^{k}$ для любого натурального $k$.

\section{СПИСОК ЛИТЕРАТУРЫ}

1. Лотов В. И., Орлова Н. Г. О числе пересечений полосы траекториями случайного блуждания. - Матем. сб., 2003, т. 194, № 6, с. 135-146.

2. Феллер В. Введение в теорию вероятностей и ее приложения, т. 1, 2. М.: Мир, 1984, 528 с.; 752 c.

3. Боровков A.A. Теория вероятностей. М: Эдиториал УРСС, 1999, 470 с.

Поступила в редакцию

21.VI.2006

Исправленный вариант

10.I.2008

\section{(c) 2008 г. ДЖАОШВИЛИ В.", ПУРТУХИЯ О.* \\ ОБОБЩЕНИЕ ФОРМУЛЫ ОКОНЕ-ХАУСМАНА-КЛАРКА ДЛЯ КОМПЕНСИРОВАННОГО ПУАССОНОВСКОГО ПРОЦЕССА ${ }^{1)}$}

Для компенсированного пуассоновского процесса определены пространства соболевского типа $D_{p, 1, \alpha}^{C P}(1 \leqslant p \leqslant 2)$ и для функционалов

* Тбилисский государственный университет им. Ив. Джавахишвили, ул. Университетская 2; Институт математики им. А. Размадзе, ул. М. Алексидзе, 1, Тбилиси 0193, Грузия, e-mail: o.purtukhia@math.sci.tsu.ge

1) Работа выполнена при финансовой поддержке Грузинского национального фонда науки, грант № 337/07, 06_223_3-104 